TRANSACTIONS OF THE

AMERICAN MATHEMATICAL SOCIETY

Volume 348, Number 7, July 1996

\title{
DIVISORS ON GENERIC COMPLETE INTERSECTIONS IN PROJECTIVE SPACE
}

\author{
GENG XU
}

\begin{abstract}
Let $V$ be a generic complete intersection of hypersurfaces of degree $d_{1}, d_{2}, \cdots, d_{m}$ in $n$-dimensional projective space. We study the question when a divisor on $V$ is nonrational or of general type, and give an alternative proof of a result of Ein. We also give some improvement of Ein's result in the case $d_{1}+d_{2}+\cdots+d_{m}=n+2$.
\end{abstract}

\section{INTRODUCTION}

Let $V$ be a generic complete intersection of hypersurfaces of degree $d_{1}, d_{2}, \cdots, d_{m}$ in $\mathbf{P}^{n}$. A conjecture of Kobayashi (cf. [L]) states that $V$ is hyperbolic if $d=$ $d_{1}+d_{2}+\cdots+d_{m} \geq n+2$. In general, $\mathrm{S}$. Lang [L] has conjectured that a variety $X$ is hyperbolic if and only if every subvariety of $X$ is of general type. In this paper, we will prove the following

Theorem 1. Let $V$ be a complete intersection of $m$ generic hypersurfaces of degree $d_{1}, d_{2}, \cdots, d_{m}$ in $\mathbf{P}^{n}, M \subset V$ a reduced and irreducible divisor, $p_{g}(M)$ the geometric genus of the desingularization of $M$. Assume that $1 \leq m \leq n-3$ and $d_{i} \geq 2$ for all $i$. Then

(1) $p_{g}(M) \geq n-1$ if $d=d_{1}+d_{2}+\cdots+d_{m} \geq n+2$,

(2) $M$ is of general type if $d=d_{1}+d_{2}+\cdots+d_{m}>n+2$.

In [E1,E2], Ein has shown that $M$ is nonrational if $d \geq n+2$, and is of general type if $d>n+2$. Here we are going to give an alternative proof of it. Ein also proved that every subvariety of $V$ of dimension $l$ is nonrational if $d \geq 2 n-m-l+1$, and is of general type if $d>2 n-m-l+1$. Therefore the improvement we made here is in the case $d=n+2$ and $l=n-m-1$. In particular, we conclude that the divisor $M$ can not be an abelian variety. If a variety $X$ is hyperbolic, then every rational map of an abelian variety or $\mathbf{P}^{1}$ into $X$ is constant. On the other hand, Lang $[\mathrm{L}]$ conjectured that this condition is also sufficient for $X$ to be hyperbolic.

If $V$ is a generic hypersurface in $\mathbf{P}^{n}$, it was first shown by Clemens [CKM] that $V$ contains no rational curves, if $\operatorname{deg} V \geq n-1$. In [X1], we study generic surfaces in $\mathbf{P}^{3}$, obtain that every curve $C$ on $S$ has geometric genus $g(C) \geq \frac{1}{2} d(d-3)-2$ $(d=\operatorname{deg} S)$, and the bound is sharp. We also obtain results about divisors on a generic hypersurface in $\mathbf{P}^{n}$. In [X2], we generalize these results to some nongeneric cases.

Received by the editors August 5, 1995.

1991 Mathematics Subject Classification. Primary 14J70, 14 B07.

Partially Supported by NSF grant DMS-9401547. 
When $V$ is a generic quintic 3 -fold in $\mathbf{P}^{4}$, a conjecture of Clemens says that $V$ should contain only finitely many rational curves of given degree, which is equivalent to the statement that every divisor on $V$ must have a nonnegative Kodaira dimension. Chang and Ran $[\mathrm{CR}]$ has proved that $V$ does not contain a reduced and irreducible divisor which admits a desingularization having a numerically effective anticanonical bundle.

To establish Theorem 1, we need to get control over the singularities of the divisor $M$ on $V$. The method we use here is deformation of singularity as we did in $[\mathrm{X} 1]$.

Throughout this paper we work over the complex number field $\mathbb{C}$.

Finally, I am very grateful to Herbert Clemens, Mark Green and Jonathan Wahl for helpful conversations.

\section{Deformation of Singularities}

For simplicity of notations, we will give a proof of Theorem 1 in the case $m=2$.

First of all, we recall some definitions from [X1].

Let $\mathrm{V}$ be an $n$-dimensional smooth variety, and $M \subset V$ be a reduced and irreducible divisor. According to Hironaka [H], there is a desingularization of $M$ :

$$
V_{m+1} \stackrel{\pi_{m+1}}{\longrightarrow} V_{m} \stackrel{\pi_{m}}{\longrightarrow} \cdots \stackrel{\pi_{2}}{\longrightarrow} V_{1} \stackrel{\pi_{1}}{\longrightarrow} V_{0}=V
$$

so that the proper transform $\tilde{M}$ of $M$ in $V_{m+1}$ is smooth. Here $V_{j} \stackrel{\pi_{j}}{\longrightarrow} V_{j-1}$ is the blow-up of $V_{j-1}$ along a $\nu_{j-1}$-dimensional submanifold $X_{j-1}$ with $E_{j-1} \subset V_{j}$ the exceptional divisor. If $X_{j-1}$ is a $\mu_{j-1}$-fold singular submanifold of the proper transform of $M$ in $V_{j-1}$, we say that $M$ has a type $\mu=\left(\mu_{j}, X_{j}, E_{j} \mid j \in\{0,1, \ldots, m\}\right)$ singularity.

If $M \subset V$ has a type $\mu=\left(\mu_{j}, X_{j}, E_{j} \mid j \in \Gamma\right)$ singularity, $\Omega \subset V$ is an open set, we localize our definition by saying that $M$ has a type $\mu_{\Omega}=\left(\mu_{j}, X_{j}, E_{j} \mid j \in \Gamma_{\Omega}=\right.$ $\left\{j \mid \exists q \in E_{j}, q\right.$, is an infinitely near point of some $\left.\left.p \in \Omega\right\}\right)$ singularity on $\Omega$.

Given any resolution of the singularity of $M \subset V$ as above, if $D \subset V$ is a divisor, such that

$$
\pi_{j}^{*}\left(\cdots\left(\pi_{2}^{*}\left(\pi_{1}^{*}(D)-\delta_{0} E_{0}\right)-\delta_{1} E_{1}\right)-\cdots\right)-\delta_{j-1} E_{j-1}
$$

is an effective divisor for all $j=1,2, \ldots, m+1$, then we say that $D$ has a weak type $\delta=\left(\delta_{j}, X_{j}, E_{j} \mid j \in\{0,1, \ldots, m\}\right)$ singularity. It is easy to see that a type $\mu$ singularity implies a weak type $\mu$ singularity.

Assume that $M \subset V$ has a type $\mu=\left(\mu_{j}, X_{j}, E_{j} \mid j \in\{0,1, \ldots, m\}\right)$ singularity. The following lemma describes the connection between the singularities of $M$ and the canonical bundle of the desingularization $\tilde{M}$ of $M$.

Lemma 2. A section of $K_{V} \otimes M$ with a weak type $\mu-1=\left(\mu_{j}-1, X_{j}, E_{j} \mid j \in\right.$ $\{0,1, \ldots, m\})$ singularity induces a section of $K_{\tilde{M}}$.

Proof. Proposition 1.1 in [X1]. $\quad$ q.e.d.

Definition. Let $T \subset \mathbb{C}^{N}$ be an open neighborhood of the origin $0 \in T$. Assuming that $\sigma: M \longrightarrow T$ is a family of reduced equidimensional algebraic varieties, $M_{t}=$ $\sigma^{-1}(t)$, then we say that the family $M_{t}$ is $\mu$-equisingular at $t=0$ in the sense 
that we can resolve the singularity of $M_{\tilde{\tau}}$ simultaneously, that is, there is a proper morphism $\pi: \tilde{M} \longrightarrow M$, so that $\sigma \circ \pi: \tilde{M} \longrightarrow T$ is a flat map and

$$
\sigma \circ \pi: \tilde{M}_{t}=(\sigma \circ \pi)^{-1}(t) \longrightarrow M_{t}
$$

is a resolution of the singularities of $M_{t}$. Moreover, if $M_{t}$ has a type $\mu(t)=$ $\left(\mu_{j}(t), X_{j}(t), E_{j}(t) \mid j \in \Gamma(t)\right)$ singularity with the above resolution, then $\mu_{j}(t)=\mu_{j}$ and $\Gamma(t)=\Gamma$ are independent of $t$, and the exceptional divisors and the singular loci of the desingularization $\tilde{M}_{t} \longrightarrow M_{t}$ have the same configuration for all $t$.

Now we state a lemma concerning the local deformation theory of singular divisors.

Lemma 3. If $M_{t}=\left\{g_{t}\left(z_{1}, \ldots, z_{n}\right)=0\right\}$ is a $\mu$-equisingular family of varieties defined in an open set $\Omega \subset \mathbb{C}^{n}$, and $M_{t}$ has a type $\mu(t)_{\Omega}=\left(\mu_{j}, X_{j}(t), E_{j}(t)\right.$ | $j \in\{0, \ldots, m\})$ singularity on $\Omega$, then the variety $\left\{\left.\frac{d g_{t}}{d t}\right|_{t=0}=0\right\}$ has a weak type $\mu(0)_{\Omega}-1=\left(\mu_{j}-1, X_{j}(0), E_{j}(0) \mid j \in\{0, \ldots, m\}\right)$ singularity on $\Omega$.

Proof. Lemma 4.4 in $[\mathrm{X} 1]$. $\quad$ q.e.d.

Let $\left\{Z_{i}\right\}$ be some homogeneous coordinates of $\mathbf{P}^{n}, F \in H^{0}\left(\mathbf{P}^{n}, \mathcal{O}(r)\right)$ and $G \in$ $H^{0}\left(\mathbf{P}^{n}, \mathcal{O}(l)\right)$ be homogeneous polynomials. We define

$$
\frac{\partial(F, G)}{\partial\left(Z_{i}, Z_{j}\right)}=\operatorname{det}\left|\begin{array}{ll}
\frac{\partial F}{\partial Z_{i}} & \frac{\partial F}{\partial Z_{j}} \\
\frac{\partial G}{\partial Z_{i}} & \frac{\partial G}{\partial Z_{j}}
\end{array}\right|
$$

The next lemma tells us how to use deformation of singularities to produce special homogeneous polynomials.

Lemma 4. Let $F_{1, t} \in H^{0}\left(\mathbf{P}^{n}, \mathcal{O}\left(d_{1}\right)\right), F_{2, t} \in H^{0}\left(\mathbf{P}^{n}, \mathcal{O}\left(d_{2}\right)\right), G_{t} \in H^{0}\left(\mathbf{P}^{n}, \mathcal{O}(k)\right)$, and $M_{t}=\left\{F_{1, t}=0\right\} \cap\left\{F_{2, t}=0\right\} \cap\left\{G_{t}=0\right\}$ be a $\mu$-equisingular family of varieties with a type $\mu(t)=\left(\mu_{j}, X_{j}(t), E_{j}(t) \mid j \in \Gamma\right)$ singularity. Setting

$$
\left.\frac{d F_{1, t}}{d t}\right|_{t=0}=F_{1}^{\prime},\left.\quad \frac{d F_{2, t}}{d t}\right|_{t=0}=F_{2}^{\prime},\left.\quad \frac{d G_{t}}{d t}\right|_{t=0}=G^{\prime},
$$

and assuming that both the varieties $\left\{F_{i, t}=0\right\}(i=1,2)$ and $\left\{F_{1, t}=0\right\} \cap\left\{F_{2, t}=0\right\}$ are smooth for $t$ in a neighborhood of 0 . Then the divisor

$$
\left\{\frac{\partial\left(F_{1,0}, F_{2,0}\right)}{\partial\left(Z_{i}, Z_{j}\right)} G^{\prime}-\frac{\partial\left(G_{0}, F_{2,0}\right)}{\partial\left(Z_{i}, Z_{j}\right)} F_{1}^{\prime}-\frac{\partial\left(F_{1,0}, G_{0}\right)}{\partial\left(Z_{i}, Z_{j}\right)} F_{2}^{\prime}=0\right\}
$$

$(i, j=0,1, \ldots, n)$ on $V=\left\{F_{1,0}=0\right\} \cap\left\{F_{2,0}=0\right\}$ has a weak type $\mu(0)-1=$ $\left(\mu_{j}-1, X_{j}(0), E_{j}(0) \mid j \in \Gamma\right)$ singularity, where $\left\{Z_{0}, Z_{1}, \cdots, Z_{n}\right\}$ are homogeneous coordinates of $\mathbf{P}^{n}$.

Proof. For any point $P \in M_{0}$, we can find an open set $\Omega \ni P$ of $V$, and generic homogeneous coordinates $\left\{Z_{i}^{\prime}\right\}$ with

$$
Z_{i}^{\prime}=\sum_{j=0}^{n} l_{i j} Z_{j} \quad(i=0,1, \ldots, n)
$$


so that

$$
\frac{\partial\left(F_{1,0}, F_{2,0}\right)}{\partial\left(Z_{i}^{\prime}, Z_{j}^{\prime}\right)} \neq 0
$$

on $\Omega$ for all $i \neq j(i, j=0,1, \cdots, n)$. Assuming $M_{0}$ has a type $\mu_{\Omega}(0)=\left(\mu_{j}, X_{j}(0)\right.$, $\left.E_{j}(0) \mid j \in \Gamma_{\Omega}\right)$ singularity on $\Omega$. Denoting

$$
\left\{z_{1}, z_{2}, \cdots, z_{n}\right\}=\left\{\frac{Z_{1}^{\prime}}{Z_{0}^{\prime}}, \frac{Z_{2}^{\prime}}{Z_{0}^{\prime}}, \cdots, \frac{Z_{n}^{\prime}}{Z_{0}^{\prime}}\right\}
$$

if we solve the equation

$$
F_{1, t}\left(1, z_{1}, z_{2}, \cdots, z_{n}\right)=0, \quad F_{2, t}\left(1, z_{1}, z_{2}, \cdots, z_{n}\right)=0
$$

near the point $P(t)$, where $P(0)=P$, and get

$$
z_{1}=\varphi_{1, t}\left(z_{3}, \cdots, z_{n}\right), \quad z_{2}=\varphi_{2, t}\left(z_{3}, \cdots, z_{n}\right),
$$

then on some open set of $\mathbb{C}^{n-2}, M_{t}$ is a $\mu$-equisingular family of divisors locally defined by the equation

$$
G_{t}\left(1, \varphi_{1, t}, \varphi_{2, t}, z_{3}, \cdots, z_{n}\right)=0 .
$$

By Lemma 3, the divisor locally defined by the equation

$$
\left.\frac{d G_{t}}{d t}\left(1, \varphi_{1, t}\left(z_{3}, \cdots, z_{n}\right), \varphi_{2, t}\left(z_{3}, \cdots, z_{n}\right), z_{3}, \cdots, z_{n}\right)\right|_{t=0}=0
$$

on $\Omega$ has a weak type $\mu_{\Omega}(0)-1=\left(\mu_{j}-1, X_{j}(0), E_{j}(0) \mid j \in \Gamma_{\Omega}\right)$ singularity.

Now a detailed computation shows that

$$
\begin{aligned}
\frac{d G_{t}}{d t} & \left.\left(1, \varphi_{1, t}, \varphi_{2, t}, z_{3}, \cdots, z_{n}\right)\right|_{t=0}=G^{\prime}+\left.\frac{\partial G_{0}}{\partial Z_{1}^{\prime}} \frac{d \varphi_{1, t}}{d t}\right|_{t=0}+\left.\frac{\partial G_{0}}{\partial Z_{2}^{\prime}} \frac{d \varphi_{2, t}}{d t}\right|_{t=0} \\
& =\left\{\frac{\partial\left(F_{1,0}, F_{2,0}\right)}{\partial\left(Z_{1}^{\prime}, Z_{2}^{\prime}\right)}\right\}^{-1}\left\{\frac{\partial\left(F_{1,0}, F_{2,0}\right)}{\partial\left(Z_{1}^{\prime}, Z_{2}^{\prime}\right)} G^{\prime}-\frac{\partial\left(G_{0}, F_{2,0}\right)}{\partial\left(Z_{1}^{\prime}, Z_{2}^{\prime}\right)} F_{1}^{\prime}-\frac{\partial\left(F_{1,0}, G_{0}\right)}{\partial\left(Z_{1}^{\prime}, Z_{2}^{\prime}\right)} F_{2}^{\prime}\right\} .
\end{aligned}
$$

Then the divisor

$$
\left\{\frac{\partial\left(F_{1,0}, F_{2,0}\right)}{\partial\left(Z_{1}^{\prime}, Z_{2}^{\prime}\right)} G^{\prime}-\frac{\partial\left(G_{0}, F_{2,0}\right)}{\partial\left(Z_{1}^{\prime}, Z_{2}^{\prime}\right)} F_{1}^{\prime}-\frac{\partial\left(F_{1,0}, G_{0}\right)}{\partial\left(Z_{1}^{\prime}, Z_{2}^{\prime}\right)} F_{2}^{\prime}=0\right\}
$$

has a weak type $\mu_{\Omega}(0)-1$ singularity on $\Omega$. Similarly, the divisor

$$
\left\{\frac{\partial\left(F_{1,0}, F_{2,0}\right)}{\partial\left(Z_{i}^{\prime}, Z_{j}^{\prime}\right)} G^{\prime}-\frac{\partial\left(G_{0}, F_{2,0}\right)}{\partial\left(Z_{i}^{\prime}, Z_{j}^{\prime}\right)} F_{1}^{\prime}-\frac{\partial\left(F_{1,0}, G_{0}\right)}{\partial\left(Z_{i}^{\prime}, Z_{j}^{\prime}\right)} F_{2}^{\prime}=0\right\} \quad(i, j=0,1, \cdots, n)
$$

has a weak type $\mu_{\Omega}(0)-1$ singularity on $\Omega$. Finally, since the expression

$$
\frac{\partial\left(F_{1,0}, F_{2,0}\right)}{\partial\left(Z_{i}, Z_{j}\right)} G^{\prime}-\frac{\partial\left(G_{0}, F_{2,0}\right)}{\partial\left(Z_{i}, Z_{j}\right)} F_{1}^{\prime}-\frac{\partial\left(F_{1,0}, G_{0}\right)}{\partial\left(Z_{i}, Z_{j}\right)} F_{2}^{\prime}
$$


is a linear combination of expressions

$$
\frac{\partial\left(F_{1,0}, F_{2,0}\right)}{\partial\left(Z_{s}^{\prime}, Z_{l}^{\prime}\right)} G^{\prime}-\frac{\partial\left(G_{0}, F_{2,0}\right)}{\partial\left(Z_{s}^{\prime}, Z_{l}^{\prime}\right)} F_{1}^{\prime}-\frac{\partial\left(F_{1,0}, G_{0}\right)}{\partial\left(Z_{s}^{\prime}, Z_{l}^{\prime}\right)} F_{2}^{\prime}, \quad(s, l=0,1, \cdots, n)
$$

and weak type $\mu_{\Omega}(0)-1$ singularity is additive (cf. section 1 in [X1]), we conclude that the divisor

$$
\left\{\frac{\partial\left(F_{1,0}, F_{2,0}\right)}{\partial\left(Z_{i}, Z_{j}\right)} G^{\prime}-\frac{\partial\left(G_{0}, F_{2,0}\right)}{\partial\left(Z_{i}, Z_{j}\right)} F_{1}^{\prime}-\frac{\partial\left(F_{1,0}, G_{0}\right)}{\partial\left(Z_{i}, Z_{j}\right)} F_{2}^{\prime}=0\right\}
$$

has a weak type $\mu_{\Omega}(0)-1$ singularity on $\Omega$, hence it has a weak type $\mu(0)-1$ singularity on $V$. q.e.d.

Remark. In general, if

$$
V_{t}=\left\{F_{1, t}=0\right\} \cap\left\{F_{2, t}=0\right\} \cap \cdots \cap\left\{F_{m, t}=0\right\}
$$

is a complete intersection of $m$ hypersurfaces, and $M_{t}^{*}=V_{t} \cap\left\{G_{t}=0\right\}$ is a $\mu$ equisingular family of divisors. Then one can state and prove an analogy of Lemma 4 with the divisor

$$
\left\{\frac{\partial\left(F_{1,0}, F_{2,0}\right)}{\partial\left(Z_{i}, Z_{j}\right)} G^{\prime}-\frac{\partial\left(G_{0}, F_{2,0}\right)}{\partial\left(Z_{i}, Z_{j}\right)} F_{1}^{\prime}-\frac{\partial\left(F_{1,0}, G_{0}\right)}{\partial\left(Z_{i}, Z_{j}\right)} F_{2}^{\prime}=0\right\}
$$

replaced by a divisor of the form

$$
\begin{aligned}
& \left\{\frac{\partial\left(F_{1,0}, F_{2,0}, F_{3,0}, \cdots, F_{m, 0}\right)}{\partial\left(Z_{i_{1}}, Z_{i_{2}}, Z_{i_{3}}, \cdots, Z_{i_{m}}\right)} G^{\prime}-\frac{\partial\left(G_{0}, F_{2,0}, F_{3,0}, \cdots, F_{m, 0}\right)}{\partial\left(Z_{i_{1}}, Z_{i_{2}}, Z_{i_{3}} \cdots, Z_{i_{m}}\right)} F_{1}^{\prime}\right. \\
& -\frac{\partial\left(F_{1,0}, G_{0}, F_{3,0}, \cdots, F_{m, 0}\right)}{\partial\left(Z_{i_{1}}, Z_{i_{2}}, Z_{i_{3}}, \cdots, Z_{i_{m}}\right)} F_{2}^{\prime}-\frac{\partial\left(F_{1,0}, F_{2,0}, G_{0}, \cdots, F_{m, 0}\right)}{\partial\left(Z_{i_{1}}, Z_{i_{2}}, Z_{i_{3}}, \cdots, Z_{i_{m}}\right)} F_{3}^{\prime} \\
& \left.-\cdots-\frac{\partial\left(F_{1,0}, F_{2,0}, F_{3,0}, \cdots, G_{0}\right)}{\partial\left(Z_{i_{1}}, Z_{i_{2}}, Z_{i_{3}}, \cdots, Z_{i_{m}}\right)} F_{m}^{\prime}=0\right\},
\end{aligned}
$$

here $i_{1}, \cdots, i_{m}=0,1, \cdots, n$.

\section{Proof of Theorem 1}

Let $V=\left\{F_{1}=0\right\} \cap\left\{F_{2}=0\right\} \subset \mathbf{P}^{n}$ be a complete intersection of generic hypersurfaces $\left\{F_{1}=0\right\}$ and $\left\{F_{2}=0\right\}$ of degree $d_{1}$ and $d_{2}$. By our assumption $m \leq n-3$, that is $\operatorname{dim} V \geq 3$, we know that Pic $V=\mathbb{Z}$ and it is generated by $\mathcal{O}_{V}(1)$, thanks to the Lefschetz theorem. Now if $M \subset V$ is a reduced and irreducible divisor, then it is a complete intersection of $V$ with another hypersurface $\{G=0\}$ of degree $k$. Here $F_{1}, F_{2}$ and $G$ are homogeneous polynomials.

Proposition 5. Let $V$ be a complete intersection of $m$ generic hypersurfaces of degree $d_{1}, d_{2}, \cdots, d_{m}$ in $\mathbf{P}^{n}$, and $M \subset V$ a reduced and irreducible divisor. Assume that $d=d_{1}+d_{2}+\cdots+d_{m} \geq n+2,1 \leq m \leq n-3$ and $d_{i} \geq 2$ for all $i$. Then there is a desingularization $\sigma: \tilde{M} \rightarrow M$ of $M$, and we have

$$
\operatorname{dim} H^{0}\left(\tilde{M}, K_{\tilde{M}} \otimes \sigma^{*} \mathcal{O}(-(d-n-2))\right) \geq n-1
$$


Remark. This is an improvement of an early result of L. Ein [E2] which states that

$$
H^{0}\left(\tilde{M}, K_{\tilde{M}} \otimes \sigma^{*} \mathcal{O}(-(d-n-2))\right) \neq 0 .
$$

Assuming Proposition 5, now we can give the

Proof of Theorem 1. (1) If $d \geq n+2$, then $H^{0}(M, \mathcal{O}(d-n-2)) \neq 0$, by Proposition 5 ,

$$
\operatorname{dim} H^{0}\left(\tilde{M}, K_{\tilde{M}} \otimes \sigma^{*} \mathcal{O}(-d+n+2)\right) \geq n-1 .
$$

Hence we have

$$
\begin{aligned}
p_{g}(M) & =\operatorname{dim} H^{0}\left(\tilde{M}, K_{\tilde{M}}\right) \\
& \geq \operatorname{dim} H^{0}\left(\tilde{M}, K_{\tilde{M}} \otimes \sigma^{*} \mathcal{O}(-d+n+2)\right)+\operatorname{dim} H^{0}\left(\tilde{M}, \sigma^{*} \mathcal{O}(d-n-2)\right)-1 \\
& \geq n-1,
\end{aligned}
$$

thanks to Hopf's theorem.

(2) If $d>n+2$, then $d-n-2 \geq 1$. From

$$
\operatorname{dim} H^{0}\left(\tilde{M}, K_{\tilde{M}} \otimes \sigma^{*} \mathcal{O}(-(d-n-2))\right) \geq n-1>0,
$$

we conclude that $M$ is of general type.

q.e.d.

We now begin the proof of Proposition 5. For simplicity of notation, we will assume that $m=2$.

Assume the contrary; namely, for any generic complete intersection of 2 hypersurfaces of degree $d_{1}, d_{2}$, there is a reduced and irreducible divisor on it with

$$
\operatorname{dim} H^{0}\left(\tilde{M}, K_{\tilde{M}} \otimes \sigma^{*} \mathcal{O}(-(d-n-2))\right)<n-1 .
$$

Set

$$
\begin{gathered}
B=\left\{\left\{F_{1}, F_{2}\right\} \in H^{0}\left(\mathbf{P}^{n}, \mathcal{O}\left(d_{1}\right)\right) \times H^{0}\left(\mathbf{P}^{n}, \mathcal{O}\left(d_{2}\right)\right) \mid\right. \text { both varieties } \\
\left.\left\{F_{i}=0\right\}(i=1,2) \text { and }\left\{F_{1}=0\right\} \cap\left\{F_{2}=0\right\} \text { are smooth }\right\}, \\
A_{k}=\left\{\left\{F_{1}, F_{2}, G\right\} \in H^{0}\left(\mathbf{P}^{n}, \mathcal{O}\left(d_{1}\right)\right) \times H^{0}\left(\mathbf{P}^{n}, \mathcal{O}\left(d_{2}\right)\right) \times H^{0}\left(\mathbf{P}^{n}, \mathcal{O}(k)\right) \mid\right. \\
\left\{F_{1}, F_{2}\right\} \in B, M=\{G=0\} \cap V \text { is a reduced and irreducible divisor on } \\
\left.V=\left\{F_{1}=0\right\} \cap\left\{F_{2}=0\right\}, \operatorname{dim} H^{0}\left(\tilde{M}, K_{\tilde{M}} \otimes \sigma^{*} \mathcal{O}(-(d-n-2))\right)<n-1\right\} .
\end{gathered}
$$

Then the map

$$
\bigcup_{k=1}^{\infty} A_{k} \rightarrow B
$$

is dominant by assumption. Hence the map $A_{k} \rightarrow B$ is dominant for some $k$. Therefore at some regular point $\left\{F_{1}, F_{2}\right\}$ of $B$, we can find a smooth section $B \rightarrow$ $A_{k}$, that is, there is a triple

$$
\left\{F_{1}, F_{2}, G\right\} \in H^{0}\left(\mathbf{P}^{n}, \mathcal{O}\left(d_{1}\right)\right) \times H^{0}\left(\mathbf{P}^{n}, \mathcal{O}\left(d_{2}\right)\right) \times H^{0}\left(\mathbf{P}^{n}, \mathcal{O}(k)\right),
$$

which has the following property: both varieties $\left\{F_{i}=0\right\}(i=1,2)$ and $V=\left\{F_{1}=\right.$ $0\} \cap\left\{F_{2}=0\right\}$ are smooth, the divisor $M=V \cap\{G=0\}$ is reduced and irreducible, 
and for any deformation $F_{1, t}$ of $F_{1}=F_{1,0}$ and $F_{2, t}$ of $F_{2}=F_{2,0}$, there is a unique deformation $G_{t}$ of $G=G_{0}$, so that the divisor

$$
M_{t}=\left\{F_{1, t}=0\right\} \cap\left\{F_{2, t}=0\right\} \cap\left\{G_{t}=0\right\}
$$

on $\left\{F_{1, t}=0\right\} \cap\left\{F_{2, t}=0\right\}$ has

$$
\operatorname{dim} H^{0}\left(\tilde{M}_{t}, K_{\tilde{M}_{t}} \otimes \sigma_{t}^{*} \mathcal{O}(-(d-n-2))\right)<n-1 .
$$

Here $\sigma_{t}^{*}: \tilde{M}_{t} \rightarrow M_{t}$ is a desingularization of $M_{t}$. Moreover, we can assume that the family $M_{t}$ is $\mu$-equisingular, and $M_{t}$ has a type $\mu(t)=\left(\mu_{j}, X_{j}(t), E_{j}(t) \mid j \in \Gamma\right)$ singularity.

Let $\left\{Z_{i}\right\}$ be fixed homogeneous coordinates of $\mathbf{P}^{n}$. By Lemma 4 , for any deformation $F_{1}^{\prime} \in H^{0}\left(\mathbf{P}^{n}, \mathcal{O}\left(d_{1}\right)\right)$ of $F_{1}$ and $F_{2}^{\prime} \in H^{0}\left(\mathbf{P}^{n}, \mathcal{O}\left(d_{2}\right)\right)$ of $F_{2}$, there is a unique deformation $G^{\prime} \in H^{0}\left(\mathbf{P}^{n}, \mathcal{O}(k)\right)$ of $G$, so that the divisor

$$
\left\{\frac{\partial\left(F_{1,0}, F_{2,0}\right)}{\partial\left(Z_{i}, Z_{j}\right)} G^{\prime}-\frac{\partial\left(G_{0}, F_{2,0}\right)}{\partial\left(Z_{i}, Z_{j}\right)} F_{1}^{\prime}-\frac{\partial\left(F_{1,0}, G_{0}\right)}{\partial\left(Z_{i}, Z_{j}\right)} F_{2}^{\prime}=0\right\} \quad(i, j=0,1, \ldots, n)
$$

on $V=\left\{F_{1,0}=0\right\} \cap\left\{F_{2,0}=0\right\}=\left\{F_{1}=0\right\} \cap\left\{F_{2}=0\right\}$ has a weak type $\mu(0)-1=\left(\mu_{j}-1, X_{j}(0), E_{j}(0) \mid j \in \Gamma\right)$ singularity. Denote $G^{\prime}=\Phi\left(F_{1}^{\prime}, F_{2}^{\prime}\right)$. Then we have a map

$$
\Phi: \quad H^{0}\left(\mathbf{P}^{n}, \mathcal{O}\left(d_{1}\right)\right) \times H^{0}\left(\mathbf{P}^{n}, \mathcal{O}\left(d_{2}\right)\right) \longrightarrow H^{0}\left(\mathbf{P}^{n}, \mathcal{O}(k)\right) /\left(F_{1}, F_{2}, G\right),
$$

here $\left(F_{1}, F_{2}, G\right)$ is the ideal generated by $F_{1}, F_{2}, G$.

Lemma 6. $\Phi$ is linear in $F_{1}, F_{2} \bmod \left(F_{1}, F_{2}, G\right)$.

Proof. Otherwise, since

$$
\begin{aligned}
& \frac{\partial\left(F_{1,0}, F_{2,0}\right)}{\partial\left(Z_{i}, Z_{j}\right)}\left(\Phi\left(a F_{1}^{\prime}+b \tilde{F}_{1}^{\prime}, F_{2}^{\prime}\right)-a \Phi\left(F_{1}^{\prime}, 0\right)-b \Phi\left(\tilde{F}_{1}^{\prime}, 0\right)-\Phi\left(0, F_{2}^{\prime}\right)\right) \\
& =\left\{\frac{\partial\left(F_{1,0}, F_{2,0}\right)}{\partial\left(Z_{i}, Z_{j}\right)} \Phi\left(a F_{1}^{\prime}+b \tilde{F}_{1}^{\prime}, F_{2}^{\prime}\right)-\frac{\partial\left(G_{0}, F_{2,0}\right)}{\partial\left(Z_{i}, Z_{j}\right)}\left(a F_{1}^{\prime}+b \tilde{F}_{1}^{\prime}\right)-\frac{\partial\left(F_{1,0}, G_{0}\right)}{\partial\left(Z_{i}, Z_{j}\right)} F_{2}^{\prime}\right\} \\
& \quad-a\left\{\frac{\partial\left(F_{1,0}, F_{2,0}\right)}{\partial\left(Z_{i}, Z_{j}\right)} \Phi\left(F_{1}^{\prime}, 0\right)-\frac{\partial\left(G_{0}, F_{2,0}\right)}{\partial\left(Z_{i}, Z_{j}\right)} F_{1}^{\prime}\right\} \\
& -b\left\{\frac{\partial\left(F_{1,0}, F_{2,0}\right)}{\partial\left(Z_{i}, Z_{j}\right)} \Phi\left(\tilde{F}_{1}^{\prime}, 0\right)-\frac{\partial\left(G_{0}, F_{2,0}\right)}{\partial\left(Z_{i}, Z_{j}\right)} \tilde{F}_{1}^{\prime}\right\} \\
& \quad-\left\{\frac{\partial\left(F_{1,0}, F_{2,0}\right)}{\partial\left(Z_{i}, Z_{j}\right)} \Phi\left(0, F_{2}^{\prime}\right)-\frac{\partial\left(F_{1,0}, G_{0}\right)}{\partial\left(Z_{i}, Z_{j}\right)} F_{2}^{\prime}\right\}
\end{aligned}
$$

and for any point $P \in V$,

$$
\frac{\partial\left(F_{1,0}, F_{2,0}\right)}{\partial\left(Z_{i}, Z_{j}\right)}(P) \neq 0
$$

for some $i, j$. By Lemma 4 and the additivity of weak type $\mu(0)-1$ singularity, the divisor

$$
\left.\left\{\Phi\left(a F_{1}^{\prime}+b \tilde{F}_{1}^{\prime}, F_{2}^{\prime}\right)-a \Phi\left(F_{1}^{\prime}, 0\right)-b \Phi\left(\tilde{F}_{1}^{\prime}, 0\right)-\Phi\left(0, F_{2}^{\prime}\right)\right)=0\right\}
$$


will have a weak type $\mu(0)-1=\left(\mu_{j}-1, X_{j}(0), E_{j}(0) \mid j \in \Gamma\right)$ singularity on $V$. On the other hand, by the adjunction formula, we have

$$
K_{V} \otimes M=\mathcal{O}(d+k-n-1) .
$$

If $\Phi$ is not linear mod $\left(F_{1}, F_{2}, G\right)$, then

$$
\Phi\left(a F_{1}^{\prime}+b \tilde{F}_{1}^{\prime}, F_{2}^{\prime}\right)-a \Phi\left(F_{1}^{\prime}, 0\right)-b \Phi\left(\tilde{F}_{1}^{\prime}, 0\right)-\Phi\left(0, F_{2}^{\prime}\right)
$$

will generate a section of $K_{\tilde{M}} \otimes \mathcal{O}(-(d-n-2)-1)$ by Lemma 2, which will imply that

$$
\operatorname{dim} H^{0}\left(\tilde{M}, K_{\tilde{M}} \otimes \sigma^{*} \mathcal{O}(-(d-n-2))\right) \geq n-1
$$

because $\operatorname{dim} H^{0}(M, \mathcal{O}(1)) \geq n-1$. Here we use the fact that $\operatorname{deg} F_{i}=d_{i} \geq 2$. q.e.d.

Let $\left\{Y_{i}\right\}$ be another homogeneous coordinate of $\mathbf{P}^{n}$. Now we take a special deformation $F_{1}^{\prime}=Y_{p} U(p=0,1, \cdots, n)$ of $F_{1}$ with $U \in H^{0}\left(\mathbf{P}^{n}, \mathcal{O}\left(d_{1}-1\right)\right)$. Since

$$
\begin{aligned}
& \frac{\partial\left(F_{1,0}, F_{2,0}\right)}{\partial\left(Z_{i}, Z_{j}\right)}\left(Y_{p} \Phi\left(Y_{q} U, 0\right)-Y_{q} \Phi\left(Y_{p} U, 0\right)\right) \\
& \quad=Y_{p}\left(\frac{\partial\left(F_{1,0}, F_{2,0}\right)}{\partial\left(Z_{i}, Z_{j}\right)} \Phi\left(Y_{q} U, 0\right)-\frac{\partial\left(G_{0}, F_{2,0}\right)}{\partial\left(Z_{i}, Z_{j}\right)} Y_{q} U\right) \\
& \quad-Y_{q}\left(\frac{\partial\left(F_{1,0}, F_{2,0}\right)}{\partial\left(Z_{i}, Z_{j}\right)} \Phi\left(Y_{p} U, 0\right)-\frac{\partial\left(G_{0}, F_{2,0}\right)}{\partial\left(Z_{i}, Z_{j}\right)} Y_{p} U\right),
\end{aligned}
$$

by Lemma 4 we conclude that the divisor $\left\{Y_{p} \Phi\left(Y_{q} U, 0\right)-Y_{q} \Phi\left(Y_{p} U, 0\right)=0\right\}$ on $V$ has a weak type $\mu(0)-1$ singularity.

Lemma 7. If $\operatorname{dim} H^{0}\left(\tilde{M}, K_{\tilde{M}} \otimes \sigma^{*} \mathcal{O}(-(d-n-2))\right)<n-1$, then there is a linear map

$$
\Phi_{1}: H^{0}\left(\mathbf{P}^{n}, \mathcal{O}\left(d_{1}-1\right)\right) \times H^{0}\left(\mathbf{P}^{n}, \mathcal{O}\left(d_{2}-1\right)\right) \rightarrow H^{0}\left(\mathbf{P}^{n}, \mathcal{O}(k-1)\right) /\left(F_{1}, F_{2}, G\right),
$$

so that for any $U \in H^{0}\left(\mathbf{P}^{n}, \mathcal{O}\left(d_{1}-1\right)\right)$, and $W \in H^{0}\left(\mathbf{P}^{n}, \mathcal{O}\left(d_{2}-1\right)\right)$, the divisor

$$
\left\{\frac{\partial\left(F_{1,0}, F_{2,0}\right)}{\partial\left(Z_{i}, Z_{j}\right)} \Phi_{1}(U, W)-\frac{\partial\left(G_{0}, F_{2,0}\right)}{\partial\left(Z_{i}, Z_{j}\right)} U-\frac{\partial\left(F_{1,0}, G_{0}\right)}{\partial\left(Z_{i}, Z_{j}\right)} W=0\right\}
$$

$(i, j=0,1, \cdots, n)$ on $V$ has a weak type $\mu(0)-1$ singularity.

Proof. Let $Y, H \in H^{0}\left(\mathbf{P}^{n}, \mathcal{O}(1)\right)$ be 2 hyperplanes, and $U \in H^{0}\left(\mathbf{P}^{n}, \mathcal{O}\left(d_{1}-1\right)\right)$ be a fixed polynomial. By the argument before Lemma 7 (choose $Y_{p}=Y, Y_{q}=H$ ), we know that the divisor $\{Y \Phi(H U, 0)-H \Phi(Y U, 0)=0\}$ on $V$ has a weak type $\mu(0)-1$ singularity. Since we have

$$
K_{V} \otimes M=\mathcal{O}(d+k-n-1),
$$

and $Y \Phi(H U, 0)-H \Phi(Y U, 0) \in H^{0}\left(\mathbf{P}^{n}, \mathcal{O}(k+1)\right)$, if

$$
Y \Phi(H U, 0)-H \Phi(Y U, 0) \not \equiv 0
$$


on $M$, that is $Y \Phi(H U, 0)-H \Phi(Y U, 0) \notin\left(F_{1}, F_{2}, G\right)$, then it will induce a section of $K_{\tilde{M}} \otimes \sigma^{*} \mathcal{O}(-(d-n-2))$ by Lemma 2. Denote

$$
\Lambda_{H}=\left\{Y \mid Y \Phi(H U, 0)-H \Phi(Y U, 0) \in\left(F_{1}, F_{2}, G\right)\right\} \subset H^{0}\left(\mathbf{P}^{n}, \mathcal{O}(1)\right) .
$$

The linearity of $\Phi$ implies that $\Lambda_{H}$ is a linear subspace of $H^{0}\left(\mathbf{P}^{n}, \mathcal{O}(1)\right)$. We conclude that $\operatorname{dim} \Lambda_{H} \geq 2$ by our assumption that

$$
\operatorname{dim} H^{0}\left(\tilde{M}, K_{\tilde{M}} \otimes \sigma^{*} \mathcal{O}(-(d-n-2))\right) \leq n-2 .
$$

Hence there is a nontrivial hyperplane $Y_{H} \in \Lambda_{H}$ such that

$$
Y_{H} \notin\left(H, F_{1}, F_{2}, G\right),
$$

thanks to the fact that $\operatorname{deg} F_{i} \geq 2$.

Let $\sigma: \tilde{M} \rightarrow M$ be a desingularization of $M$. Then the linear system $\left|\sigma^{*} \mathcal{O}(1)\right|$ on $\tilde{M}$ is base point free. Since $\operatorname{dim} M=\operatorname{dim} V-1 \geq 2$, and $M$ is reduced and irreducible, Bertini's theorem implies that the generic hyperplane section of $\tilde{M}$ is irreducible. Therefore we can choose a generic hyperplane $H$, so that $H \cap M$ is irreducible and reduced. By our construction of $Y_{H}$, we have

$$
Y_{H} \Phi(H U, 0)-H \Phi\left(Y_{H} U, 0\right) \in\left(F_{1}, F_{2}, G\right),
$$

that is $Y_{H} \Phi(H U, 0) \in\left(H, F_{1}, F_{2}, G\right)$. The fact that $Y_{H} \notin\left(H, F_{1}, F_{2}, G\right)$ and that $H \cap M$ is irreducible now gives us $\Phi(H U, 0) \in\left(H, F_{1}, F_{2}, G\right)$. Therefore,

$$
\Phi(H U, 0)=H U^{*} \bmod \left(F_{1}, F_{2}, G\right)
$$

for some $U^{*} \in H^{0}\left(\mathbf{P}^{n}, \mathcal{O}(k-1)\right)$, and $U^{*}$ is unique $\bmod \left(F_{1}, F_{2}, G\right)$ because $M$ is reduced and irreducible. Similarly, for any $W \in H^{0}\left(\mathbf{P}^{n}, \mathcal{O}\left(d_{2}-1\right)\right)$, there is a $W^{*} \in H^{0}\left(\mathbf{P}^{n}, \mathcal{O}(k-1)\right)$, such that

$$
\Phi(0, H W)=H W^{*} \bmod \left(F_{1}, F_{2}, G\right) .
$$

Now we define

$$
\Phi_{1}(U, W)=U^{*}+W^{*} \in H^{0}\left(\mathbf{P}^{n}, \mathcal{O}(k-1)\right) /\left(F_{1}, F_{2}, G\right),
$$

then $\Phi_{1}$ is independent of the choice of the generic hyperplane $H$.

From Lemma 4, we know that the divisor

$$
\left\{\frac{\partial\left(F_{1,0}, F_{2,0}\right)}{\partial\left(Z_{i}, Z_{j}\right)} \Phi(H U, H W)-\frac{\partial\left(G_{0}, F_{2,0}\right)}{\partial\left(Z_{i}, Z_{j}\right)} H U-\frac{\partial\left(F_{1,0}, G_{0}\right)}{\partial\left(Z_{i}, Z_{j}\right)} H W=0\right\}
$$

on $V$ has a weak type $\mu(0)-1$ singularity. Using the fact that

$$
\Phi(H U, H W)=\Phi(H U, 0)+\Phi(0, H W)=H \Phi_{1}(U, W) \bmod \left(F_{1}, F_{2}, G\right),
$$

we find that the divisor

$$
\left\{H\left(\frac{\partial\left(F_{1,0}, F_{2,0}\right)}{\partial\left(Z_{i}, Z_{j}\right)} \Phi_{1}(U, W)-\frac{\partial\left(G_{0}, F_{2,0}\right)}{\partial\left(Z_{i}, Z_{j}\right)} U-\frac{\partial\left(F_{1,0}, G_{0}\right)}{\partial\left(Z_{i}, Z_{j}\right)} W\right)=0\right\}
$$


on $V$ has a weak type $\mu(0)-1$ singularity. Therefore we know that the divisor

$$
\left\{\frac{\partial\left(F_{1,0}, F_{2,0}\right)}{\partial\left(Z_{i}, Z_{j}\right)} \Phi_{1}(U, W)-\frac{\partial\left(G_{0}, F_{2,0}\right)}{\partial\left(Z_{i}, Z_{j}\right)} U-\frac{\partial\left(F_{1,0}, G_{0}\right)}{\partial\left(Z_{i}, Z_{j}\right)} W=0\right\}
$$

on $V$ has a weak type $\mu(0)-1$ singularity if we choose the generic hyperplane $H$ such that it is in general position with respect to the singular locus of $M$. Again, we may assume that $\Phi_{1}$ to be linear $\bmod \left(F_{1}, F_{2}, G\right)$ as we did for $\Phi$. q.e.d.

We continue the proof of Theorem 1. If

$$
\operatorname{dim} H^{0}\left(\tilde{M}, K_{\tilde{M}} \otimes \sigma^{*} \mathcal{O}(-(d-n-2))\right)<n-1,
$$

we can repeat the argument in the proof of Lemma 7 again on the triple

$$
\left(U, W, \Phi_{1}(U, W)\right) \in H^{0}\left(\mathbf{P}^{n}, \mathcal{O}\left(d_{1}-1\right)\right) \times H^{0}\left(\mathbf{P}^{n}, \mathcal{O}\left(d_{2}-1\right)\right) \times H^{0}\left(\mathbf{P}^{n}, \mathcal{O}(k-1)\right)
$$

instead of the triple

$$
\left(F_{1}^{\prime}, F_{2}^{\prime}, \Phi\left(F_{1}^{\prime}, F_{2}^{\prime}\right)\right) \in H^{0}\left(\mathbf{P}^{n}, \mathcal{O}\left(d_{1}\right)\right) \times H^{0}\left(\mathbf{P}^{n}, \mathcal{O}\left(d_{2}\right)\right) \times H^{0}\left(\mathbf{P}^{n}, \mathcal{O}(k)\right),
$$

and using Lemma 7 instead of Lemma 4. After repeating this process for several times, eventually we arrive at the following situation.

Case (1). $d_{1} \leq k$ and $d_{2} \leq k$. There are

$$
R_{i j} \in H^{0}\left(\mathbf{P}^{n}, \mathcal{O}\left(k-d_{1}\right)\right) \text { and } S_{i j} \in H^{0}\left(\mathbf{P}^{n}, \mathcal{O}\left(k-d_{2}\right)\right),
$$

so that both the divisor

$$
\left\{\frac{\partial\left(F_{1,0}, F_{2,0}\right)}{\partial\left(Z_{i}, Z_{j}\right)} R_{i j}-\frac{\partial\left(G_{0}, F_{2,0}\right)}{\partial\left(Z_{i}, Z_{j}\right)} \cdot 1=0\right\}
$$

and the divisor

$$
\left\{\frac{\partial\left(F_{1,0}, F_{2,0}\right)}{\partial\left(Z_{i}, Z_{j}\right)} S_{i j}-\frac{\partial\left(F_{1,0}, G_{0}\right)}{\partial\left(Z_{i}, Z_{j}\right)} \cdot 1=0\right\}
$$

on $V$ have weak type $\mu(0)-1$ singularities. Moreover,

$$
R_{i j} \equiv R, S_{i j} \equiv S \bmod \left(F_{1}, F_{2}, G\right)
$$

are independent of $i, j$, because we assume that the deformation $G^{\prime}=\Phi\left(F_{1}^{\prime}, F_{2}^{\prime}\right)$ is unique for given $F_{1}^{\prime}, F_{2}^{\prime}$ (the reason is the same as we assume that $\Phi$ is linear).

Consider the following linear equation

$$
\begin{aligned}
& \alpha \frac{\partial F_{1,0}}{\partial Z_{i}}+\beta \frac{\partial F_{2,0}}{\partial Z_{i}}=\frac{\partial G_{0}}{\partial Z_{i}}-\frac{\partial F_{1,0}}{\partial Z_{i}} R-\frac{\partial F_{2,0}}{\partial Z_{i}} S \\
& \alpha \frac{\partial F_{1,0}}{\partial Z_{j}}+\beta \frac{\partial F_{2,0}}{\partial Z_{j}}=\frac{\partial G_{0}}{\partial Z_{j}}-\frac{\partial F_{1,0}}{\partial Z_{j}} R-\frac{\partial F_{2,0}}{\partial Z_{j}} S .
\end{aligned}
$$

When we solve this equation, we get

$$
\begin{aligned}
& \frac{\partial\left(F_{1,0}, F_{2,0}\right)}{\partial\left(Z_{i}, Z_{j}\right)} \alpha=\frac{\partial\left(G_{0}, F_{2,0}\right)}{\partial\left(Z_{i}, Z_{j}\right)} \cdot 1-\frac{\partial\left(F_{1,0}, F_{2,0}\right)}{\partial\left(Z_{i}, Z_{j}\right)} R, \\
& \frac{\partial\left(F_{1,0}, F_{2,0}\right)}{\partial\left(Z_{i}, Z_{j}\right)} \beta=\frac{\partial\left(F_{1,0}, G_{0}\right)}{\partial\left(Z_{i}, Z_{j}\right)} \cdot 1-\frac{\partial\left(F_{1,0}, F_{2,0}\right)}{\partial\left(Z_{i}, Z_{j}\right)} S .
\end{aligned}
$$


Hence the divisor

$$
\left\{\frac{\partial\left(F_{1,0}, F_{2,0}\right)}{\partial\left(Z_{i}, Z_{j}\right)}\left(\frac{\partial G_{0}}{\partial Z_{i}}-\frac{\partial F_{1,0}}{\partial Z_{i}} R-\frac{\partial F_{2,0}}{\partial Z_{i}} S\right)=0\right\}
$$

on $V$ has a weak type $\mu(0)-1$ singularity. For any point $P \in V$, we can choose generic homogeneous coordinates so that

$$
\frac{\partial\left(F_{1,0}, F_{2,0}\right)}{\partial\left(Z_{i}, Z_{j}\right)} \neq 0
$$

near $P$ for all $i \neq j$. Then the divisor

$$
\left\{\frac{\partial G}{\partial Z_{i}}-\frac{\partial F_{1}}{\partial Z_{i}} R-\frac{\partial F_{2}}{\partial Z_{i}} S=0\right\}=\left\{\frac{\partial G_{0}}{\partial Z_{i}}-\frac{\partial F_{1,0}}{\partial Z_{i}} R-\frac{\partial F_{2,0}}{\partial Z_{i}} S=0\right\}
$$

has a weak type $\mu(0)-1$ singularity in a neighborhood of $P$. Now let $\left\{Y_{i}\right\}$ be another homogeneous coordinate of $\mathbf{P}^{n}$. Since

$$
\frac{\partial G}{\partial Y_{j}}-\frac{\partial F_{1}}{\partial Y_{j}} R-\frac{\partial F_{2}}{\partial Y_{j}} S
$$

is a linear combination of expressions

$$
\frac{\partial G}{\partial Z_{i}}-\frac{\partial F_{1}}{\partial Z_{i}} R-\frac{\partial F_{2}}{\partial Z_{i}} S \quad(i=0,1, \cdots, n)
$$

and weak type $\mu(0)-1$ singularity is additive, we conclude that the divisor

$$
\left\{\frac{\partial G}{\partial Y_{j}}-\frac{\partial F_{1}}{\partial Y_{j}} R-\frac{\partial F_{2}}{\partial Y_{j}} S=0\right\}
$$

on $V$ has a weak type $\mu(0)-1$ singularity. If

$$
\frac{\partial G}{\partial Y_{j}}-\frac{\partial F_{1}}{\partial Y_{j}} R-\frac{\partial F_{2}}{\partial Y_{j}} S=0 \quad \bmod \left(F_{1}, F_{2}, G\right)
$$

for all $j$, then

$$
\frac{\partial G}{\partial Y_{j}}-\frac{\partial F_{1}}{\partial Y_{j}} R-\frac{\partial F_{2}}{\partial Y_{j}} S=0 \quad \bmod \left(F_{1}, F_{2}\right)
$$

because it's degree $k-1<k$, and the Euler equation will imply that $G \in\left(F_{1}, F_{2}\right)$, which is impossible.

Therefore

$$
\frac{\partial G}{\partial Y_{j}}-\frac{\partial F_{1}}{\partial Y_{j}} R-\frac{\partial F_{2}}{\partial Y_{j}} S \notin\left(F_{1}, F_{2}, G\right)
$$

for some $j$, that is,

$$
\frac{\partial G}{\partial Y_{j}}-\frac{\partial F_{1}}{\partial Y_{j}} R-\frac{\partial F_{2}}{\partial Y_{j}} S \not \equiv 0
$$

on $M$. Now we can choose

$$
H_{1}, \cdots, H_{n-1} \in H^{0}\left(\mathbf{P}^{n}, \mathcal{O}(1)\right) \text {, }
$$


so that $H_{i}$ generates a linear subspace of dimension $n-1$ and $G$ is not there (in case $\operatorname{deg} G=1$ ). Then

$$
\left(\frac{\partial G}{\partial Y_{j}}-\frac{\partial F_{1}}{\partial Y_{j}} R-\frac{\partial F_{2}}{\partial Y_{j}} S\right) H_{1} H_{i} \quad(i=1,2, \cdots, n-1)
$$

will induce $n-1$ linear independent sections of $K_{\tilde{M}} \otimes \sigma^{*} \mathcal{O}(-(d-n-2))$ by Lemma 2. A contradiction.

Case (2). $d_{1} \leq k<d_{2}$. Then Lemma 7 implies that the divisor

$$
\left\{\frac{\partial\left(F_{1,0}, G_{0}\right)}{\partial\left(Z_{i}, Z_{j}\right)}=0\right\}
$$

on $V$ has a weak type $\mu(0)-1$ singularity. The argument in case (1) (take $S=0$ ) shows that for some $j$, the divisor

$$
\left\{\frac{\partial G}{\partial Y_{j}}-\frac{\partial F_{1}}{\partial Y_{j}} R=0\right\}
$$

on $V$ has a weak type $\mu(0)-1$ singularity, and it is nontrivial on $M$. Again we get

$$
\operatorname{dim} H^{0}\left(\tilde{M}, K_{\tilde{M}} \otimes \sigma^{*} \mathcal{O}(-(d-n-2))\right) \geq n-1 .
$$

Case (3). $d_{1}>k$ and $d_{2}>k$. This time, we conclude that both divisors

$$
\left\{\frac{\partial\left(F_{1,0}, G_{0}\right)}{\partial\left(Z_{i}, Z_{j}\right)}=0\right\} \quad \text { and } \quad\left\{\frac{\partial\left(G_{0}, F_{2,0}\right)}{\partial\left(Z_{i}, Z_{j}\right)}=0\right\}
$$

on $V$ have weak type $\mu(0)-1$ singularities. The argument in case (1) (take $R=$ $S=0)$ shows that for some $j$, the divisor $\left\{\frac{\partial G}{\partial Y_{j}}=0\right\}$ on $V$ has a weak type $\mu(0)-1$ singularity, and it is nontrivial on $M$. We conclude again that

$$
\operatorname{dim} H^{0}\left(\tilde{M}, K_{\tilde{M}} \otimes \sigma^{*} \mathcal{O}(-(d-n-2))\right) \geq n-1 .
$$

This completes the proof of Proposition 7 .

\section{REFERENCES}

[CKM] H. Clemens, J. Kóllar and S. Mori, Higher Dimensional Complex Geometry, Asterisque, 1988. MR 90j: 14046

[CR] M.C. Chang and Z. Ran, Divisors on some generic hypersurfaces, J. Diff. Geom. 38 (1993), 671-678. MR 95e:14031

[E1] L. Ein, Subvarieties of generic complete intersection, Invent. Math. 94 (1988), 163-169. MR 89i: 14002

[E2] L. Ein, Subvarieties of generic complete intersections II, Math. Ann. 289 (1991), no. 3, 465-471. MR 92h:14002

$[\mathrm{H}] \quad$ H. Hironaka, Resolution of singularities of an algebraic variety over a field of characteristic zero, Annals of Math. 79 (1964), 109-203, 205-326. MR 33:7333

[L] S. Lang, Hyperbolic and diophantine analysis, Bull. of A.M.S. 14 (1986), 159-205. MR 87h:32051

[X1] G. Xu, Subvarieties of general hypersurfaces in projective space, J. Diff. Geom. 39 (1994), 139-172. MR 95d:14043

[X2] G. Xu, Divisors on hypersurfaces, Math. Zeitschrift 219 (1995), 581-589.

Department of Mathematics, Johns Hopkins University, Baltimore, Maryland 21218

E-mail address: geng@math.jhu.edu 